\title{
Wettability of clay surfaces and its implication on the status of water in unsaturated porous media
}

\author{
LOÏC GUÉRIN ${ }^{1}$, LAURENT MICHOT ${ }^{1}$, ERIC FERRAGE ${ }^{2}$, \\ FRANCIS CLARET $^{3}$ AND VIRGINIE MARRY ${ }^{4}$ \\ ${ }^{1}$ Université Pierre et Marie Curie (UPMC) \\ ${ }^{2}$ Université de Poitiers \\ ${ }^{3}$ BRGM \\ ${ }^{4}$ Sorbonne Université, UPMC Univ Paris 06, UMR 8234 \\ Presenting Author: loic-ou@hotmail.fr
}

The wide use of clay minerals in industrial applications is partly due to their remarkable properties of water retention at the mineral surface. While clays have been extensively studied under saturated conditions, studies in unsaturated media remain rare. However, in the context of radioactive waste storage in deep geological formation, partially saturated transient conditions appear during the operation phase of the storage areas for high-level radioactive waste and after the closure phase by thermal effect and by the release of $\mathrm{H} 2$ gas by radiolysis.

In order to provide a multiscale vision of the mechanisms of water and solutes diffusion, from the scale of the clay layer to that of the stacking of clay particles under various conditions of partial water saturation, understanding the physical and chemical processes at the origin of the liquid / gas surface tension and the contact angle at the solid / liquid / gas interface within unsaturated nanoporous clayey materials is necessary. It is especially important to better understand the role of the crystallochemistry of clay minerals and the nature of the charge-compensating cations on the surfaces wettability.

Molecular simulations have the advantage of offering a detailed view of the physicochemistry of these systems at the atomic level, but they rely on the reliability of the force field used to describe interatomic interactions. In this study we perform angle experiments in solid/water/air and solid/oil/water interfaces on cleaved clays surfaces with different crystallographic structures and counter-ions [1-2]. Clays were chosen according to the location of their negative charge (tetrahedral layer or octahedral layer), their hydroxyde or fluorine content, and according to whether or not single-crystal exist. Experimental contact angles are compared to those obtained by molecular dynamic simulations of a water drop on clay surfaces, with the commonly used forcefield ClayFF and the polarisable forcefield PIM (Polarizable Ion Model) currently being developped in the PHENIX laboratory [3].

\section{References}

[1] Rotenberg et all (2011), JACS 133, 20521-20527.

[2] Ballah, Chamerois, Durand-Vidal, Malikova, Levitz and Michot (2016), Colloids and Surfaces A 511, 255-263.

[3] Tesson, Louisfrema, Salanne, Boutin, Ferrage, Rotenberg and Marry (2018), J. Phys. Chem. C 122, 24690-24704. 